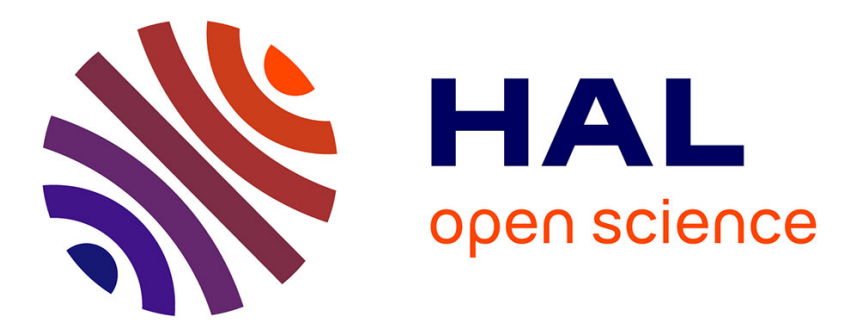

\title{
Les universitaires de Mai 68: tensions structurelles et radicalisation syndicale autour de la réforme du Comité consultatif des universités
}

Emmanuelle Picard

\section{- To cite this version:}

Emmanuelle Picard. Les universitaires de Mai 68: tensions structurelles et radicalisation syndicale autour de la réforme du Comité consultatif des universités. Benoit Bruno, Chevandier Christian, Morin Gilles, Richard Gilles, Vergnon Gilles. À chacun son Mai? Le tour de France de mai-juin 1968, Presses universitaires de Rennes, pp.269-280, 2011. halshs-00593723

\section{HAL Id: halshs-00593723 \\ https://shs.hal.science/halshs-00593723}

Submitted on 17 May 2011

HAL is a multi-disciplinary open access archive for the deposit and dissemination of scientific research documents, whether they are published or not. The documents may come from teaching and research institutions in France or abroad, or from public or private research centers.
L'archive ouverte pluridisciplinaire HAL, est destinée au dépôt et à la diffusion de documents scientifiques de niveau recherche, publiés ou non, émanant des établissements d'enseignement et de recherche français ou étrangers, des laboratoires publics ou privés. 


\title{
Les universitaires de Mai 68 : tensions structurelles et radicalisation syndicale autour de la réforme du Comité consultatif des universités
}

\author{
Emmanuelle PICARD
}

\begin{abstract}
Dans l'histoire de la profession universitaire, les années 1960 constituent un moment charnière. Après un siècle et demi d'histoire linéaire, caractérisée par l'accumulation des textes réglementaires et l'affirmation progressive de leur autonomie, dans un contexte de faible croissance numérique, les universitaires français doivent faire face à un bouleversement brutal, consécutif à l'extraordinaire croissance des effectifs étudiants. L'augmentation rapide du nombre des universitaires s'accompagne en effer d'une modification importante de la morphologie du corps professionnel, dont les effets sont lisibles dans la radicalisation des prises de position politiques et syndicales consécutives aux " événements de Mai " et à leurs conséquences.

L'analyse des débats du début des années 1970 au sujet de la principale instance de gestion du corps universitaire qu'est le comité consultatif des universités est l'occasion de mettre en évidence les tensions nouvelles qui traversent le corps universitaire et les arguments des parties en présence. Se croisent alors le temps long des structures et des pratiques héritées et le temps court des transformations induites par les nouvelles configurations professionnelles.
\end{abstract}

\section{Un groupe professionnel en pleine transformation}

Si les enjeux propres à l'organisation de l'espace académique deviennent plus aigus dans années 1970, c'est parce qu'ils s'inscrivent dorénavant dans une nouvelle configuration professionnelle. Les transformations quantitatives et morphologique du corps universitaire français entre 1960 et 1975 sont bien connues. Elles se traduisent à la fois par la plus forte augmentation numérique (on passe d'environ 6500 universitaires en 1960 à près de 30000 quinze ans plus tard, soit une multiplication par 4,5) jamais obser- 
vée depuis la création des facultés par Napoléon en 1808, et par l'inversion du rapport traditionnel entre enseignants de rang professoral (professeurs et maîtres de conférences) et enseignants subalternes, comme les montrent les données présentées dans les tableaux ci-dessous.

\begin{tabular}{|l|c|c|c|}
\hline & $\begin{array}{l}\text { Professeurs d'université } \\
\text { et maîtres de conférences }\end{array}$ & $\begin{array}{l}\text { Maîtres } \\
\text { assistants }\end{array}$ & $\begin{array}{l}\text { Assistants agrégés } \\
\text { et non agrégés }\end{array}$ \\
\hline $\mathbf{1 9 6 0}$ & 3352 & 500 & 2580 \\
\hline $\mathbf{1 9 6 8}$ & 6105 & 5426 & 8087 \\
\hline $\mathbf{1 9 7 3}$ & 9118 & 9842 & 10748 \\
\hline Augmentation & $272 \%$ & $2000 \%$ & $420 \%$ \\
\hline
\end{tabular}

3. Rapporr $\mathrm{DE}$ BAECUE, La situatian dies personnels enseignants des sniversités. Eléments de reffexion pour une reforme, Paris, La Documentation française, 1974

Evolution du nombre des enseignants (1960-1973).

Cette augmentation brutale fait suite à une période de lente croissance : les universitaires, tous statuts confondus, n'étaient encore qu'environ 6000 au début des années $1950^{\prime}$. Il s'agissait alors essentiellement de professeurs titulaires (de chaire, sans chaire, à titre personnel) et de maitres de conférences, recrutés après un doctorar (dont la forme s'apparentait au doctorat d'État); à leurs côtés, on trouvait des chargés de cours ou des chargés d'enseignement, non titulaires, qui poursuivaient l'achèvement de leur propre thèse ${ }^{2}$. Les restrictions budgétaires de l'entre-deux-guerres avaient eu pour effet de limiter la croissance du nombre des titulaires et de prendre l'habitude de recourir plus fréquemment aux vacataires, à tel point que l'administration finit par créer une catégorie spécifique : les assistants ${ }^{3}$. Il ne s'agissait pas véritablement d'un corps mais plutôt d'une désignation générale, qui recouvrait de fait des réalités très variées selon les facultés, la seule constante étant la position subordonnée. En effet, coexistaient des assistants titulaires (principalement en lettres, et qui étaient en fait titulaires d'une agrégation du second degré) et non titulaires. Selon les facultés, les conditions d'exercice et de pérennité dans le poste étaient très variables. Il n'en restait pas moins qu'une position d'assistant était vécue comme la première étape d'une carrière universitaire. Dans la pratique, les professeurs

1. Cf. Antoine Prost, Histoire génémle de l'enseignenient et de l'éducation en France, t. IV, L'école et la famille dans une société en mutation (depuis 1930) Paris, Perrin, 2004.

2. Une des seules téférences dont nous disposions actueliemenc sur les transformations strucrurelles du corps universitaire est l'article déjà ancien de Françoise Maýur, "Lévolution des corps universitaires, $1877-1968$ ", dans Christophe CharLe et Régine Ferré, Le personnel de l'enseignement superiezur anx xw et $x x^{\circ}$ siedles. CNRS, 1985, p. 11-26.

3. Ils apparaissent en 1925 dans les facultés des sciences, en 1942 dans celles des letures er dans les années 1950 dans les facultés de droit. 
recrutaient ainsi leurs élèves, contribuant à organiser une gestion étroite des modalités de renouvellement du corps professoral.

Depuis les années 1950, le système français d'enseignement supérieur avait fait l'objet d'un certain nombre de critiques, dénonçant en particulier le caractère obsolète des modalités d'accès à la carrière. La thèse française apparaissait comme très spécifique au regard des pratiques académiques des autres pays occidentaux, et semblait de plus en plus inappropriée, en particulier dans les facultés des sciences. Ce furent les premières à accueillir une nouvelle forme de diplôme doctoral, la thèse de troisième cycle en $1954^{4}$, suivies par les facultés des lettres en $1958^{5}$. Cette thèse courte devait permettre un accès anticipé à de véritables postes de titulaires, ce qui fut fait avec la création du corps des mâtrres assistants en $1960^{6}$. Il devenait dès lors possible de recruter une nouvelle catégorie d'enseignants, dont la mission principale serait d'assurer les travaux dirigés. Depuis la fin des années cinquante, l'augmentation continue du nombre des étudiants rendait nécessaire une croissance parallèle de celui des enseignants; et l'existence de corps subalternes (moins onéreux en salaires et constituant du fait de leurs services plus lourds une importante réserve horaire) a permis d'y faire face. Mais la conséquence en a été le progressif affaiblissement de la part du corps à rang professoral dans l'ensemble du corps enseignant.

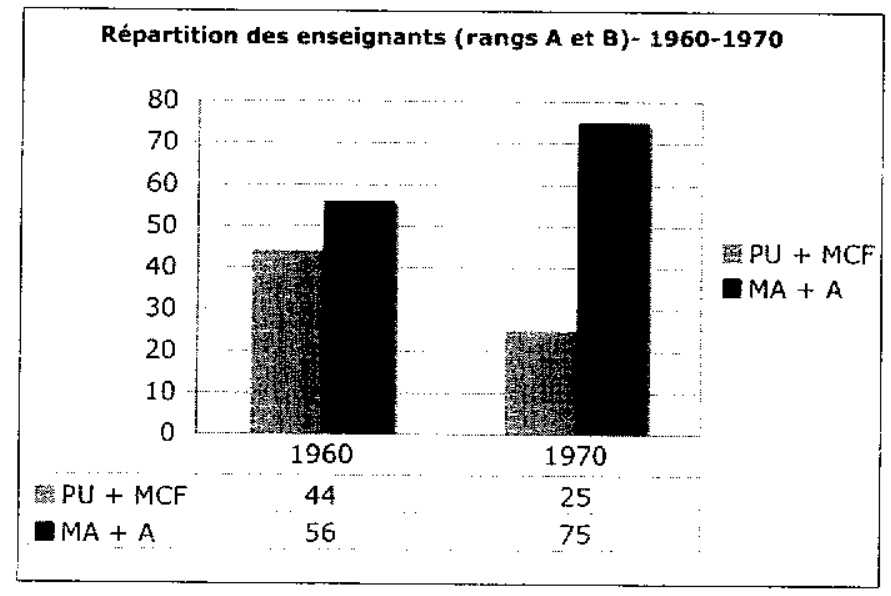

4. Décret $\mathrm{n}^{\circ}$ 54-770 portant création d'un troisième cycie dans les facultés des sciences, 20 juilles 1954.

5. Décret sur la création du diplóme de docteur sanctionnant le 3 r cycle de l'enseignement supérieur des lertres, 19 avril 1958.

6. Décret $\mathrm{n}^{\circ}$ 60-1027 sur le statut particulier des maîtres assistants des facułtés des sciences, des facultés des lettres et sciences humaines et autres établissements d'enseignement supéricur, 26 septembre 1960. 
Cette introduction massive de nouveaux enseignants n’a pas été sans conséquence sur les modalités de recrutement. Pour faire face à la nécessité, les élèves traditionnellement "élus " pour entrer dans la carrière n'étaient plus assez nombreux, et il devint nécessaire d'élargir les bases de la population d'origine. Les nouveaux entrants sont, en majorité, moins dotés en titre scolaire que leurs prédécesseurs traditionnels, même dans les disciplines les plus haut placées dans la hiérarchie universitaire : par exemple en lettres, on voit apparaitre en nombre les normaliens de Saint-Cloud ainsi que des agrégés non normaliens, mais aussi des recrues plus âgées qui se trouvaient alors dans l'enseignement secondaire La baisse des titres scolaires s'accompagne aussi pour des disciplines moins cotées d'une augmentation du recrutement féminin et d'une baisse de la moyenne de l'âge d'entrée dans l'enseignement supérieur ${ }^{?}$.

Ces nouveaux profils entrés en masse contribuent à modifier sensiblement les caractéristiques de l'ensemble de la population enseignante mais entraînent surtout un clivage accentué entre deux types de profil. La première distinction se fait en matière d'âge : au début des années 1970 , le groupe composé par les professeurs a une moyenne d'âge qui tourne autour de 50 ans (plus âgés dans les facultés de médecine, plus jeunes dans les facultés de droit et de sciences économiques); pour les maîtres de conférences, la moyenne se situe autour de 45 ans. Les maitres assistants et les assistants sont nettement plus jeunes, avec des moyennes oscillants entre 30 et 40 ans selon les matières. La seconde distinction concerne les pratiques professionnelles : aux titulaires de rang professoral, la charge des cours magistraux, des directions de recherche et d'équipe et surtout la participation aux instances de décision des facultés (conseils); aux assistants et maitres assistants, celle des travaux en petits groupes et très souvent de la correction des nombreuses copies. Les charges d'enseignement varient également : de trois heures par semaine pour les premiers, à 4 à 6 séances hebdomadaires (sans précisions horaires) pour les seconds. Pour ces derniers, la position subordonnée est très clairement exprimée dans les textes les régissant.

Pour autant, les maîtres assistants et assistants ne composent pas une population homogène. Par-delà les différences de statuts (entre les deux corps mais aussi entre les facultés), deux groupes coexistent. Le premier, le plus nombreux, est composé de ces nouvelles recrues, au profil décrit plus haut; mais subsiste également une catégorie qui s’apparente étroitement aux élus traditionnels. En effer, le mode de recrutement des élèves des professeurs en poste ne s'est pas tari et les normaliens et agrégés continuent d'intégrer ces positions subalternes qui constituent le mode d'entrée classique dans la carrière. On ne peut donc schématiser simplement le clivage qui

7. Cf. Picre Bourdieu, Homo Academicts, Les Éditions de Mintit, 1984. 
traverse la profession, par la simple distinction entre deux niveaux hiérarchiques. Plus subtilement, il convient de distinguer d'une part une carégorie dont le mode de fonctionnement, le rapport à l'activité professionnelle, aux modes de régulation du corps (recrutement, carrière...) s'inscrivent dans l'héritage séculaire (il s'agit en grande majorité des professeurs d'université et des maittres de conférences, auxquels s'ajoure la part des maîtres assistants et des assistants qui, détenteurs de caractéristiques communes, auraient été recrutés de toute façon); et une catégorie plus jeune, plus féminisée, moins dotée scolairement, entrée massivement dans les postes subalternes et moins prête à jouer le jeu traditionnel de l'institution. Ainsi, la description faire par un assistant de la faculté des lettres de Paris du " gouffre exist[ant] actuellement entre la plèbe (assistants, maîtres-assistants) et les sénateurs (maîtres de conférences, professeurs) ${ }^{8}$ " n'est vraie que partiellement. C'est ce qui explique que les attitudes face aux événements de Mai 68 ne se calquent pas exactement sur cette opposition. À Nanterre, par exemple, on trouve des partisans et des opposants au mouvement étudiant dans chacune des deux catégories, même si la proportion respective des professeurs, maitres assistants et assistants n'est cependant pas identique? . Pour autant, l'analyse de la répartition des différentes organisations syndicales fait apparaître des groupes bien identifiés, dont les prises de position seront très étroitement corrélées avec la place qu'ils occupent dans l'espace universitaire : le syndicat autonome particulièrement bien représenté dans les facultés de médecine et de sciences, est aussi celui qui rassemble le plus grand nombre de membres du corps professoral ; à l'inverse, le SNESup compte dans ses rangs près de $80 \%$ d'assistants et de maitres assistants en $1966^{10}$.

L'une des façons d'étudier les registres de discours politiques et les projets professionnels antagonistes dont ces groupes peuvent être porteurs est de s'intéresser aux discussions qui se développent à la fin des années soixante autour des transformations nécessaires des instances de régulation professionnelles que sont les sections du Comité consultatif des universités.

\section{Une négociation corporatiste}

Le clivage s'organise à la fois entre les corps et entre les facultés et fait apparaittre la coexistence de deux modèles académiques antagonistes : d'une part un modèle facultaire, en lettres et en droit, largement arc-bouté sur une hiérarchie stricte et une grande rigidité organisationnelle qui entend

8. "Faire l'Universiré. Dossier pour la réforme de l'enseignement supéricur ", Esprit, n 328 , maijuin 1964, P. 814.

9. Cf. Charles Mercier, "René Rémond à Nanterre en 68 ", Vingtième Siècle, n" 104, 2009. p. 144 .

10. Marc-Olivier Dérlaude, "Une mobilisation contre-révolutionnaire : la refondation du syndicat autonome des enseignants de médecine en mai 1968 et sa lutte pour la "sélection" "Sociétés contemporaines, $\mathbf{n}^{\circ} 73,2009$, p. $21-45$. 
préserver l'organisation traditionnelle; d'autre part, des facultés des sciences, qui dénoncent l'obsolescence d'un tel mode de fonctionnement et appellent à des transformations profondes ${ }^{11}$. Par ailleurs, les prises de position syndicales, qui sont étroitement corrélées avec le statut majoritaire de leurs membres, se surajoutent à cette partition. Les débats qui suivent Mai 68 sont l'occasion, par la radicalisation des positions qu'ils entraînent, d'observer les arguments mis en cuvre par ces différentes carégories.

Les sujets d'opposition sont nombreux : au cour des discussions, la rigidité du découpage faculcaire (qui limite le recrutement des professeurs d'un ordre de faculté à ses propres docteurs ${ }^{12}$ ), l'immobilisme qu'induit l'existence des chaires, les inégalités des positions de rang professoral (titulaire de chaire, sans chaire, à titre personnel); mais aussi les découpages rigides entre disciplines, au sein d'une instance comme le Comité consultatif des Universités, er donc la question de la place des nouvelles disciplines; ou encore le rôle des maîtres assistants et des assistants dans les décisions au sein des instances. L'ensemble de ces questions, et quelques autres, sera au cœur des propositions réformatrices d'une direction de l'enseignement supérieur dirigée par Pierre Aigrain entre 1965 et février 1968, l'un des promoteurs du colloque de Caen organisé en 1966 sur la modernisation de l'enseignement supérieur ${ }^{13}$.

L'un des principaux point d'achoppement au sein de la communauté universitaire porte sur les assistants et les maîtres assistants, et plus particulièrement leur place dans les processus de décision. L'analyse des débats autour de la nécessaire réforme du comité consultatif des universités, qui débutent en 1967 pour s'achever par une série de décrets au début des années soixante-dix, constitue un lieu très favorable à l'observation de cette tension spécifique ${ }^{14}$. La profession universitaire est, depuis la fin du XIX ${ }^{e}$ siècle, organisée autour d'un mode de gestion centralisé; pour accéder aux postes, il est nécessaire d'être inscrit sur une liste d'aptitude dont la composition est arrêtée par des représentants des différentes disciplines, réunis en section d'un Comité consultatif des univcrsités; ce sont elles

11. Il exisce bien súr des dissidences au seiı des facultés, et l'on trouve des conservateurs en sciences comme des contestataires en letrres, ainsi que nous le verrons dans les exemples proposés dans la suite de cet article.

12. Dans un entretien avec le journal Le Monde du 3 août 1967, Pierre Aigrain déclare: "L'un des problèmes par exemple : pour faire partie du conseil de faculté il faut être docreur de l'ordre de faculté concerné; un mathémaricien docteur ès sciences qui enscigne les mathémariques appliquées dans unc faculte de droit et d'économie est exclu du conscil."

13. Sur le site "Hisroire du CNRS ", une intervicw de Pierre Aigrain qui permet de se faire une idée des conditions de fonctionnement de l'administration de la recherche et de l'enseignement supérieut dans les années soixante : http://picardpl.ivry.cnrs.fr/Aigrain.hrml.

14. Les dossiers de ces débats sont consultables au Centre des archives contempotaines, à Fontainebleau. Ils ont été déposés par la direction de l'enscignement supérieur : CAC, 1977-0496, articles 7 à 10 . 
également qui sont en charge des promotions ${ }^{15}$. Cette instance est organisée sur un mode disciplinaire et facultaire rigide (quatre divisions, correspondant aux quatre ordres de facultés, décomposées en sections par discipline), et inscrite dans la hiérarchie facultaire (le doyen de la faculté se prononce, lors des promotions, sur la personne qu'il est question de promouvoir). La part des élus de rang professoral y est écrasante, renforcée par un système d'élection uninominale qui sappuie sur la réputation er la position dans le champ universitaire. Ce système posait déjà de nombreux problèmes au début des années soixante : comment intégrer les nouvelles disciplines? comment gérer la masse des maîtres assistants et leurs revendications à être partie prenante du système? comment prendre mieux en compte les activités de recherche ${ }^{16}$ ?

S'ensuit, à partir de 1965, une série de consultations organisées par la direction de l'enseignement supérieur sur les modalités de la réforme envisagée. Il est intéressant de souligner que, durant cette première période, la parole est très individualisée : ce sont des doyens, des professeurs occupant des chaires prestigieuses, plus que des syndicats ou des associations qui sont sollicités et surtout qui répondent aux propositions de la direction de l'enseignement supérieur. Le clivage déjà évoqué, sciences vs droit-lettres, joue alors à plein. Les représentants des facultés des sciences poussent à une réforme profonde, et demandent en particulier la disparition du découpage en divisions (c'est-à-dire, en fait, en facultés). Cette suppression leur semble le seul moyen de permettre la réorganisation disciplinaire nécessaire à la prise en compre des évolutions de la science contemporaine. Ils sont également favorables à la présence de chercheurs au sein des sections du comité consultatif des universités et à une meilleure représentativité des maîtres assistants. À l'inverse, les littéraires et les juristes se montrent farouchement opposés à la disparition du découpage facultaire et à la participation des chercheurs ${ }^{17}$; mais plus que tout, ils s'émeuvent du fait que les maîtres assistants et assistants, mieux représentés, puissent jouer un rôle quelconque dans les carrières des maîtres de conférences et des professeurs. Cette première réforme, discutée lors de l'année 1967, n'aboutira pas, les décrets n'ayant pas été présentés en conseil des ministres quand éclate Mai 68.

15. C'est le nom qu'il prend en 1945, mais ce mode de fonctionnement existe en fait depuis les années 1890 ; cf. Emmanuelle PICARD, "Éléments d'histoire des instances d'évaluation des enseignants chercheurs", dans Marie-Françoise Fave-Bonnet (dir.), l'évaluation dans lenseignement superieu. en question, Paris, L'Harmattan, 2010.

16. Une letre de la FEN à la direction de l'enseignement supérieur. en décembre 1961, listait déjà unc parrie de ces problèmes : "Laccroissement dú personnel enseignan des faculrés des sciences ainsi que la diversificarion de płus en plus grande des disciplines scientifiques liće d'ailleurs à l'évolution et aux progrès de la science rendent le comité consultatif inadapré à son rôle dans sa forme actuclle " (CAC, 1977-0496, article 8).

17. Il faut taire une exceprion pour Jcan-Louis Quermonne, professeur de droit qui sera llun des ardents soutiens de la réforme proposér. 
La Loi Faure du 12 novembre 1968 modifie considérablement le paysage universitaire et rend caduque une partie des discussions précédentes. Désormais, les chaires et les facultés n'existant plus, le découpage en divisions du comité consultatif des universités a vécu. Les sections disciplinaires vont constituer autant d'éléments isolés et juxtaposés, dont la réunion par groupes se fera sans lien avec les constitutions d'UER et d'universités alors en cours. Par ailleurs, les décrets d'application prévoient la réorganisation du corps enseignant en trois catégories : A pour les professeurs et les maitres de conférences; B pour les maîtres assistants er assistants du second degré (docteurs); et $\mathrm{C}$ pour les assistants non agrégés et chargés de cours complémentaires. Dans l'esprit des administrateurs de la direction de P'enseignement supérieur, le regroupement des différents personnels au sein d'une même catégorie semble conduire à une gestion commune de leur sort : ainsi, les maittres de conférences seraient juges dans des questions concernant les professeurs et les assistants de celles des maitres assistants. Mais cette utopie égalitaire ne semble pas avoir survécu plus de quelques semaines et les nouveaux projets envisagent tout au contraire de restreindre la place des subalternes en attribuant seulement un quart des places aux maîtres assistants, assistants et chefs de travaux, au motif que leur donner la parité entraînerait la formation de divisions trop nombreuses, car on ne peut pas " diminuer corrélarivement le nombre des professeurs et des maitres de conférences déjà réduit au minimum dans certaines disciplines ${ }^{18}$ ".

S'engage une nouvelle série de discussions qui conduiront à l'adoption d'un nouveau décret en $1972^{19}$. À la différence de la période précédente, les syndicats deviennent des interlocuteurs de premier plan, soit directement par le biais d'échanges entre la direction de l'enseignement supérieur et leurs directions nationales, soit par l'expression, au sein d'instances diverses, de motions portées par les syndicats. Les deux principaux points du débat portent sur la participation des membres de rang $B$ aux décisions concernant les membres de rang A; et sur la transformation des modalités d'élection de ses membres.

Ce second point, qui émane essentiellement des revendications du SNESup et du SGEN ${ }^{20}$, vise à mettre fin à des pratiques volontiers qualifiées de clientélistes ou de mandarinales, qui seraient le corollaire inévitable d'un scrutin uninominal. Pour y remédier, il est nécessaire que les élections se fassent par scrutin de liste; à l'inverse, le syndicat autonome, en particulier, dénonce les risques de démagogie électoraliste que certe

18. Nore de la DES à l'attention du ministre, 25 novembre 1968, signée Jean Sirinelli (CAC, 1977 0496 , article 9 ).

19. Décret $n^{\circ} 72-1016$ relatif au comité consultatif des universités, 6 novembre 1972

20. Le SGEN a une position moins tranchée que le SNESup et s'accommode d'une élection au scruti de liste pour le seul collège B (lettre au ministre, 20 décembre 1971): position qui est aussi celle du syndicat autonone en 1972 (lettre au ministre, 29 mars 1972) [les deux : CAC, 1977-0496. article 10]. 
option ferait courir. En 1972, le conseil de l'université Aix-Marseille 1 "émet un vœu "sur les élections au comité consultatif des universités : "Le scrutin uninominal en vigueur ne permet pas une représentation équitable de tous les courants scientifiques, ni de toutes les méthodes de recherche; il favorise la pérennité des monopoles sciencifiques; il ne permet pas aux candidats de se regrouper sur la base d'un programme ou d'un choix d'intentions sur lesquels les électeurs seraient appelés à se prononcer ${ }^{21}$." Jean Bastié, secrétaire général du syndicat autonome, fait une analyse tout à fait inverse et dénonce les dangers du scrutin "syndical ": "Car il ne faut pas perdre de vue que les partisans de la politisation de l'université ont changé de tactique depuis trois ans, leur entreprise de mainmise totalitaire sur notre Université ne repose plus pour l'instant sur l'agitation, mais sur une pénétration sournoise et souterraine des instances chargées de prendre des décisions et notamment celles relatives au personnel ${ }^{22}$. " Position partagée par la direction de l'enseignement supérieur qui indique dans une note au cabinet qu'elle a le "souci de voir les électeurs se déterminer en fonction de critères personnels et non pas sur des critères d'appartenance syndicale et politique ${ }^{23}$ ". Le décret de novembre 1972 a tranché en faveur de la position conservatrice puisque les membres des deux collèges sont toujours élus au scrutin uninominal à deux tours ${ }^{24}$, et c'est encore le cas dans le décret instaurant, en remplacement du comité consultatif, un conseil supérieur des corps universitaires en $1979^{25}$.

Lautre point de litige porte sur la présence de personnels de rang $B$ dans les instances de délibération concernant les personnels de rang $A$, mais aussi, de façon plus générale, sur l'idée d'une gestion commune par l'ensemble des représentants des personnels, tous corps confondus. C'est ainsi que le conseil transitoire de gestion de la faculté de lettres et de sciences humaines de Besançon, «considérant que dans un certain nombre de cas, les maîtres assistants et assistants siégeaient dans des sections avant ce nouveau comité consultatif, que cela convenait bien en particulier en sciences, que l'administration n'est jamais intervenue sur cette question, exprime sa désapprobation devant ce qui est ressenti comme un recul en terme de "démocratisation de la gestion des personnels" ". Pour lui, loin de " consister une pression préjudiciable à la liberté des délibérations, [certe présence] est le moyen indispensable pour que s'instaure une coopération

21. CAC, 1977-0496, article 10 .

22. Lertre de J. Bastié à la direcrion de l'enseignement supérieur. 1972 (CAC, 1977-0496, article 10).

23. Note à l'atcention de M. Rufenacht, conseiller technique au cabinet du ministre, 23/3/1972 (CAC, 1977-0496, article 10).

24. Article 5 du décret $n^{\circ} 72-1016$.

25. Décret $n^{\circ} 79-684$ relatif au Conseil supérieur des corps universitaires et modifiant le décrec $n^{\circ} 77-963$ du 24 août 1977 relatif aux commissions de spécialistes des établissements publics à caractère scientifique er culturel, 9 août 1979 . 
efficace entre les diverses catégories d'enseignants-chercheurs. Depuis deux ans, une telle collaboration est de règle dans le cadre des UER. [...] Pour toutes ces raisons, le conseil transitoire de gestion de la faculté des lettres et sciences humaines de Besançon demande instamment à la direction des Enseignements supérieurs de revenir sur le veto qu'elle veut d'opposer la présence des assistants et maîtres-assistants à l'ensemble des délibérations des sections et groupes de sections du CCU ${ }^{26}$ ". C'est aussi ce que réclamera le conseil de l'université d'Aix-Marseille 1, en 1972, dans son vœu sur l'avenir du comité consultatif :

"Force est de constater, par ailleurs, que les décisions de certaines sections du comité consultatif dans leur composition actuelle, en marière d'inscription sur les listes d'apritude, reflètent surtout des préoccupations malthusiennes, et parfois fort rétrogrades concernant l'avenir des disciplines en cause (barrage renforcé au niveau de l'inscription sur les listes, évolution freinée vers une plus grande scientificité, réticences envers des disciplines nouvelles). Ces pratiques s'appuient sur un fonctionnement non démocratique ou contestable de ces sections, en particulier sur le refus de laisser les représentants des catégories $\mathrm{B}$ et $\mathrm{C}$ assister à toutes les délibérations. [...] Parallèlement, il arrive que s'exercent, en matière de nomination et de recrutement des enseignants-chercheurs, des considérations étrangères aux seuls critères scientifiques et pédagogiques : népotisme, intervention de perites chapelles, favoritisme, critères politiques ou idéologiques. Cette situation, en l'absence d'instance nationale de recours pour les nominations, peut aboutir à la sclérose sciencifique ou pédagogique de cercaines UER, en même cemps qu'elle favorise toutes les injustices. "

L'argument développé par les partisans d'une implication plus grande des élus de rang $B$ dans les instances gestionnaires s'appuie sur différents précédents : dans un certain nombre de sections de sciences dites " dures", au sein du comité consultatif, la pratique s'était développée depuis le début des années soixante d'une forme de travail collaboratif entre tous les membres, quel que soit leur statut; par ailleurs, au sein des UER nouvellement créés, la cogestion existait dans un certain nombre de lieux. Mais il $s$ inscrit aussi directement dans les approches participatives mises en ouvre après 1968 : il s'agit d'accroître la démocratie et la transparence dans l'une des plus importantes instances de gestion des personnels enseignants.

Les opposants sont nombreux, et sans surprise se recrutent essentiellement chez les professeurs. Au nombre de leurs arguments, on retrouve la dénonciation d'une politisation des débats académiques. Dans une note

26. Motion établie lors de la séance du 10 juin 1970. à destination de la direction de l'enseignement supérieur (CAC, 1977-0496, article 1 i). 
destinée au Premier ministre 27 , Tessier, professeur à la faculté des sciences de Marseille explique :

"Au nom de la nécessité proclamée de dépasser la loi d'orientation, ces élus [SNESup] se sont engagés, dans leur programme, à faire participer à toutes les délibérations du comité toutes les catégories qui y sont représentées. Autrement dit, les assistants seraient appelés à débattre du recrutement des professeurs et, à la limite, à voter pour ce recrutement. [...] Lors de ces réunions [des sections], et conformément à une tactique éprouvée, la question de la participation à tous les débats a été soulevée par les assistants. Il apparaît que, dans plusieurs sections, la chose aurair été admise, avec plus ou moins de netreté et d'enthousiasme. Une telle manière de procéder, si elle avait lieu, serait en violation flagrante avec le titre VI de la loi d'orientation. [...] On pourrait redouter en effet, vu le monolithisme du comportement du SNESup et ses tendances politiques, que ne soient désormais retenus que les candidats présentés ou soutenus par ce syndicat et dont la couleur politique serait jugée convenable, en ne considérant la valeur politique que comme élément accessoire. [...] En résumé, il est nécessaire d'empêcher cette pratique. Elle apparait comme illégale et contraire à divers règlements d'administration $[\ldots]$, elle apparât d'autre part comme dangereuse pour la valeur du recrutement et comme favorisant le noyautage politico-syndical. "

La direction de l'enseignement supérieur transmet le dossier pour avis au Conseil d'Érat. En septembre 1970, celui-ci réaffirme le principe selon lequel ne peuvent participer aux délibérations que les membres dont "le rang est au moins égal à celui que le candidat doit obtenir ou occupe après son recrutement \%. Il précise ensuite que les membres d'un rang inférieur ne doivent pas, au risque d'entrainer la nullité de la décision, ne serait-ce qu'assister aux débats, que par leur seule présence ils pourraient influencer. S'appuyant sur cet arrêt, le directeur de l'enseignement supérieur, Jean Sirinelli, envoie une lettre aux présidents et vice-présidents du comité consultatif : "En conséquence, il y a lieu de remarquer notamment que lors de l'établissement des différentes listes d'aptitude les représentants de la catégorie à laquelle appartient le candidat avant la nomination à laquelle la liste concernée donne vocation, ne peuvent ni participer, ni même simplement assister aux délibérations. Il est précisé que le non-respect de ces règles vicie la procédure. En conséquence, toutes les nominations et les promotions prononcées après les délibérations qui seraient intervenues irrégulièrement seraient entachées d'illégalité ${ }^{28}$."

27. 19 juin 1970, note de Tessiet, professeur a la faculte des sciences de Marseille sur le fonctionnement du Comité consultarif des universités, adressée au Premier ministre et transmis au ministère de l'Éducation nationale (CAC, 1977-0496, arricle 10).

28. Letre de J. Sirinelti, le 22 seprembre 1970 (CAC, 1977-0496, arricle 10), qui a souligné " d'illègalicé ". 
28

Si les événements de Mai 68 ont introduit des nouvelles revendications de la part d'une partie du personnel enseignant, ils ont aussi induit un raidissement des différentes positions. À la lecture des archives, on a le sentiment que les projets réformateurs d'avant 1968 auraient pu permettre une transformation en douceur des instances de gestion des personnels mais que la radicalisation qui suit les "événements " a contribué à la victoire des positions les plus conservatrices. Cependant, les discussions vives autour de la place et des prérogatives des différentes catégories enseignantes ont permis que la question des staruts des universitaires émerge dans l'espace public. Elle sera au cœur des débats dans les années 1970, et aboutira aux décrets de juin 1984. 